\title{
Original Article \\ Iyengar Yoga versus Enhanced Usual Care on Blood Pressure in Patients with Prehypertension to Stage I Hypertension: a Randomized Controlled Trial
}

\author{
Debbie L. Cohen, ${ }^{1}$ LeAnne T. Bloedon, ${ }^{2}$ Rand L. Rothman, ${ }^{2}$ \\ John T. Farrar, ${ }^{3}$ Mary Lou Galantino, ${ }^{3,4}$ Sheri Volger, ${ }^{2}$ Christine Mayor, ${ }^{2}$ \\ Phillipe O. Szapary, ${ }^{2,3}$ and Raymond R. Townsend ${ }^{1}$ \\ ${ }^{1}$ Renal Division, University of Pennsylvania School of Medicine, Philadelphia, PA 19104, USA \\ ${ }^{2}$ Division of General Internal Medicine, University of Pennsylvania School of Medicine, Philadelphia, PA 19104, USA \\ ${ }^{3}$ Center for Clinical Epidemiology and Biostatistics, University of Pennsylvania, Philadelphia, PA 19104, USA \\ ${ }^{4}$ Program in Physical Therapy, Richard Stockton College of New Jersey, Pomona, NJ 08240, USA
}

Correspondence should be addressed to Debbie L. Cohen, cohendl@mail.med.upenn.edu

Received 13 October 2008; Accepted 30 July 2009

Copyright (C) 2011 Debbie L. Cohen et al. This is an open access article distributed under the Creative Commons Attribution License, which permits unrestricted use, distribution, and reproduction in any medium, provided the original work is properly cited.

The prevalence of prehypertension and Stage 1 hypertension continues to increase despite being amenable to non-pharmacologic interventions. Iyengar yoga (IY) has been purported to reduce blood pressure (BP) though evidence from randomized trials is lacking. We conducted a randomized controlled trial to assess the effects of 12 weeks of IY versus enhanced usual care (EUC) (based on individual dietary adjustment) on 24-h ambulatory BP in yoga-naïve adults with untreated prehypertension or Stage 1 hypertension. In total, 26 and 31 subjects in the IY and EUC arms, respectively, completed the study. There were no differences in $\mathrm{BP}$ between the groups at 6 and 12 weeks. In the EUC group, 24-h systolic BP (SBP), diastolic BP (DBP) and mean arterial pressure (MAP) significantly decreased by 5,3 and $3 \mathrm{mmHg}$, respectively, from baseline at 6 weeks $(P<.05)$, but were no longer significant at 12 weeks. In the IY group, $24 \mathrm{~h} \mathrm{SBP}$ was reduced by $6 \mathrm{mmHg}$ at 12 weeks compared to baseline $(P=.05) .24 \mathrm{~h} \mathrm{DBP}(P<.01)$ and MAP $(P<.05)$ decreased significantly each by $5 \mathrm{mmHg}$. No differences were observed in catecholamine or cortisol metabolism to explain the decrease in BP in the IY group at 12 weeks. Twelve weeks of IY produces clinically meaningful improvements in $24 \mathrm{~h}$ SBP and DBP. Larger studies are needed to establish the long term efficacy, acceptability, utility and potential mechanisms of IY to control BP.

\section{Introduction}

Hypertension remains a major public health issue with the latest data from the National Health and Nutrition Examination Survey (NHANES) revealing that 65 million adults in USA have hypertension [1]. Hypertension is associated with increased risks of stroke, cardiovascular disease and chronic kidney disease [2]. Patients with high normal blood pressure (BP) [systolic BP (SBP) $130-139 \mathrm{mmHg}$ or diastolic BP (DBP) $80-89 \mathrm{mmHg}$ ] fall into the category of prehypertensive [3] and are at an increased risk for adverse cardiovascular events compared with normotensive controls [4]. Lifestyle modifications (LSM) are recommended as first line approach for both prehypertensive and Stage 1 hypertension patients (SBP $140-159 \mathrm{mmHg}$ or DBP $90-99 \mathrm{mmHg}$ ) [5]. LSM include weight loss, dietary recommendations and increased physical activity which can reduce SBP by $2-$ $20 \mathrm{mmHg}$ [3], but are often difficult to sustain [6].

There is emerging data that mind-body therapies (MBTs) may be useful in managing modest elevations in BP [7]. Yoga, a movement-based MBT, is especially attractive as a candidate therapy in the management of elevated BP because of its wide appeal to Westerners and its combination of gentle physical activity, slowed regulated breathing and meditation. If yoga practice were to be effective in preventing, delaying or treating mild hypertension, this could translate into a clinical 
and health economic benefit in people motivated enough to pursue this kind of treatment. There are several reports in the literature on the effects of various yoga programs on BP [8-16]; however, most of these are case series or studies with methodological limitations.

The objective of this randomized controlled trial was to rigorously evaluate the cardiovascular and physiologic effects of a 12-week structured Iyengar yoga (IY) program compared with an enhanced usual care (EUC) intervention emphasizing dietary approaches on reducing average SBP as measured by 24-h ambulatory BP monitoring (ABPM) in adults with untreated prehypertension to Stage I hypertension.

\section{Methods}

2.1. Participants. Participants included 78 yoga-naïve adults between the ages of 22 and 69 years with untreated $\mathrm{SBP} \geq 130 \mathrm{mmHg}$ but $<160 \mathrm{mmHg}$, and DBP $<100 \mathrm{mmHg}$. Major exclusion criteria included: pregnancy or postpartum $<3$ months; current use of any medications or dietary supplements known to affect BP; body mass index (BMI) $>40 \mathrm{~kg} / \mathrm{m}^{2}$; practice of IY in previous 12 months, or active practice of any other MBT more than two times per month; diabetes mellitus; cardiovascular disease; autonomic neuropathy; current tobacco use; renal insufficiency; and $>10$ alcoholic drinks per week in women and $>15$ drinks in men. Subjects were recruited by flyers placed in the hospital and university campus and by advertisements placed in local papers and online advertising using craigslist. The University of Pennsylvania Institutional Review Board approved the trial, and all subjects provided written, informed consent. The trial was registered at the Clinical Trials website: NCT00328666.

2.2. Study Protocol. Screening BP and heart rate (HR) were measured in the morning after a $12-\mathrm{h}$ fast using a Datascope Accutorr Plus machine with an appropriate sized cuff after individuals were seated for $5 \mathrm{~min}$. Three readings were obtained, separated by 1-min intervals and the average of these readings determined eligibility. Eligible subjects returned to the General Clinical Research Center (GCRC) for an inpatient stay at Weeks 0, 6 and 12 for ABPM which was recorded over $24 \mathrm{~h}$ using Spacelabs model 90207 monitors [17]. Subjects remained in the GCRC during the inpatient stay and were sedentary. Data was determined to be satisfactory if there were at least 48 (80\%) acceptable readings (SBP between 70 and $280 \mathrm{mmHg}$ and DBP between 40 and $150 \mathrm{mmHg}$ ) between $6 \mathrm{a} . \mathrm{m}$. and 12:00 midnight, and six acceptable readings between midnight and 6 a.m. If any three consecutive readings averaged $>180 \mathrm{mmHg}$ for SBP, or $>110 \mathrm{mmHg}$ for DBP, the participant was discontinued.

2.3. Intervention. Over 23 months, seven cohorts containing 8-12 participants per cohort were recruited sequentially and randomized to either EUC or IY after completing the week 0 visit and prior to discharge from their first GCRC overnight stay. Participants randomized to IY attended twice weekly
TABLE 1: IY class structure and components.

\begin{tabular}{|c|c|}
\hline Component ${ }^{(\mathrm{a})}$ & Time \\
\hline $\begin{array}{l}\text { Instructor introduction and } \\
\text { organizational issues }\end{array}$ & $5 \mathrm{~min}$ \\
\hline Set up and take attendance & $5 \mathrm{~min}$ \\
\hline \multicolumn{2}{|l|}{ Asanas $^{(a)}$} \\
\hline Savasana (corpse pose) & $5 \mathrm{~min}$ \\
\hline Cross bolsters & $5 \mathrm{~min}$ \\
\hline $\begin{array}{l}\text { Supta baddha konasana (supine bound } \\
\text { angle pose) }\end{array}$ & $5 \mathrm{~min}$ \\
\hline $\begin{array}{l}\text { Supta swastikasana (supine fortunate } \\
\text { pose) (a) }\end{array}$ & $5 \mathrm{~min} / \mathrm{side}$ \\
\hline Bharadvajasana (a twisting pose) & $3 \times 30$ s/side \\
\hline Pavannamuktasana (release of wind pose) & $5 \mathrm{~min}$ \\
\hline $\begin{array}{l}\text { Adho mukha virasana (downward facing } \\
\text { hero pose) }\end{array}$ & $5 \mathrm{~min}$ \\
\hline $\begin{array}{l}\text { Adho mukha swastikasana (downward } \\
\text { facing fortunate pose) }\end{array}$ & $1 \mathrm{~min} /$ side \\
\hline $\begin{array}{l}\text { Adho mukha svanasana (downward } \\
\text { facing dog) }\end{array}$ & $1 \mathrm{~min}$ \\
\hline Uttanasana (standing forward bend) & $1 \mathrm{~min}$ \\
\hline $\begin{array}{l}\text { Janu sirsasana (seated forward bent with } \\
\text { bent leg) }\end{array}$ & $1 \mathrm{~min} / \mathrm{side}$ \\
\hline $\begin{array}{l}\text { Upavisthakonasana (seated forward bend } \\
\text { with wide legs) }\end{array}$ & $3 \mathrm{~min}$ \\
\hline Paschimottanasana (full forward bend) ${ }^{(a)}$ & $1 \mathrm{~min}$ \\
\hline Savasana (corpse pose) $)^{(a)}$ & $5 \mathrm{~min}$ \\
\hline Ujjayi (the conqueror) pranayama ${ }^{(a)}$ & $5 \mathrm{~min}$ \\
\hline \multicolumn{2}{|l|}{ End of class attendance ${ }^{(a)}$} \\
\hline $\begin{array}{l}\text { Total class time (including } 1 \mathrm{~min} \\
\text { transitions) }\end{array}$ & $\sim 71 \mathrm{~min}$ \\
\hline
\end{tabular}

(a) Included in the home program.

70 min IY classes for the first 6 weeks (first class was within 7 days after week 0 visit) and then once a week for the following 6 weeks. Classes were not open to the public and were taught by two IY-certified instructors. In each class, 2-10 participants were led through a sequence of adjusting the body into timed postures (asanas) and breathing techniques (pranayama) using props. The IY program (Table 1) was developed by IY-certified instructor, Joan White, with input from IY founder B.K.S. Iyengar and included specific postures that were easy to adapt for older, obese populations. During the second-half of the program (Weeks 6-12), IY participants also participated in home practice using a 25min DVD. Each participant kept a home diary to document frequency and duration of the home practice. Participants who missed three consecutive or four total IY studio classes were discontinued. Participants randomized to IY did not receive any instructions or guidance regarding LSM.

Participants randomized to EUC classes attended four 1-h group classes that met during Weeks 1, 2, 3 and 8 with 30-min individual phone contact at Weeks 5 and 10. Classes were taught by the same registered dietitian (S.V.). The classes were designed to include motivational and 
behavioral components educating participants about LSM to reduce elevated $\mathrm{BP}$ including weight reduction/maintenance, sodium reduction, alcohol restriction and increasing intake of fruits, vegetables and low-fat, calcium-rich foods. EUC participants who missed more than one of the first three classes were discontinued.

At each visit, body weight was measured on a calibrated scale. Subjects were instructed to record all food and beverages consumed for three assigned days prior to Weeks 0,6 and 12. Data was analyzed using Nutrition Data Systems for Research software, version 4.05, University of Minnesota.

In order to assess the effect of IY on the hypothalamicadrenal axis, we measured salivary cortisol and several urinary and blood biomarkers. Caffeine, alcohol and exercise were discouraged $8 \mathrm{~h}$ prior to collection. Salivary cortisol samples were collected between 7 a.m. and 8 a.m. and sealed into a container until analyzed by an ELISA assay (Salimetrics). Urine was collected over $24 \mathrm{~h}$ at each visit and stored at $4{ }^{\circ} \mathrm{C}$ during collection and transferred to a $-70^{\circ} \mathrm{C}$ freezer until analyzed. Blood was obtained after a 12-h fast at Weeks 0, 6 and 12. Urine cortisol was measured after extracting with dichloromethane using a radioimmunoassay kit (Siemen Medical Solutions Diagnostic, Los Angeles, CA, USA) Urine sodium and potassium were measured using a Laboratory Instrument 943 flame photometer. Plasma Aldosterone was also measured using the radioimmunoassay kit (Siemen Medical Solutions Diagnostic). Plasma Renin was measured by IRMA using a kit by Diagnostic Systems Laboratories, Webster, TX, USA. Plasma Metanephrines were measured by ELISA using a kit by ImmunoBiological Laboratories, MN55432.

The following psychometric evaluations were performed at Weeks 0,6 and 12 to determine the effect of IY on mood, anxiety, stress and health-related quality of life as compared with EUC. The specific tests performed included the Profile of Mood States (POMS), the Perceived Stress Survey (PSS) and the Short-form Health Survey (SF-36). Research staff manually scored the completed questionnaires and the results were entered into the database.

\subsection{Statistics}

2.4.1. Sample Size. All sample size calculations were done and verified using Power and Precision version 2.04 (Dataxiom Software Inc.), which estimated that with a $15 \%$ dropout rate, we would need to randomize 30 subjects per group. The sample size was based on an $80 \%$ power calculation to detect a $4 \mathrm{mmHg}$ SBP difference between groups.

2.4.2. Statistical Analysis. Differences in baseline characteristics between subjects who completed and those who dropped out were tested using $t$-tests for continuous variables and chi-squared tests for categorical variables. Outcomes within the IY group and within the EUC group were tested by comparing means at baseline to means at Week 6 and means at Week 12 using $t$-tests on the equality of means. Outcomes for the IY group relative to the EUC group were tested by comparing the difference in means between baseline and

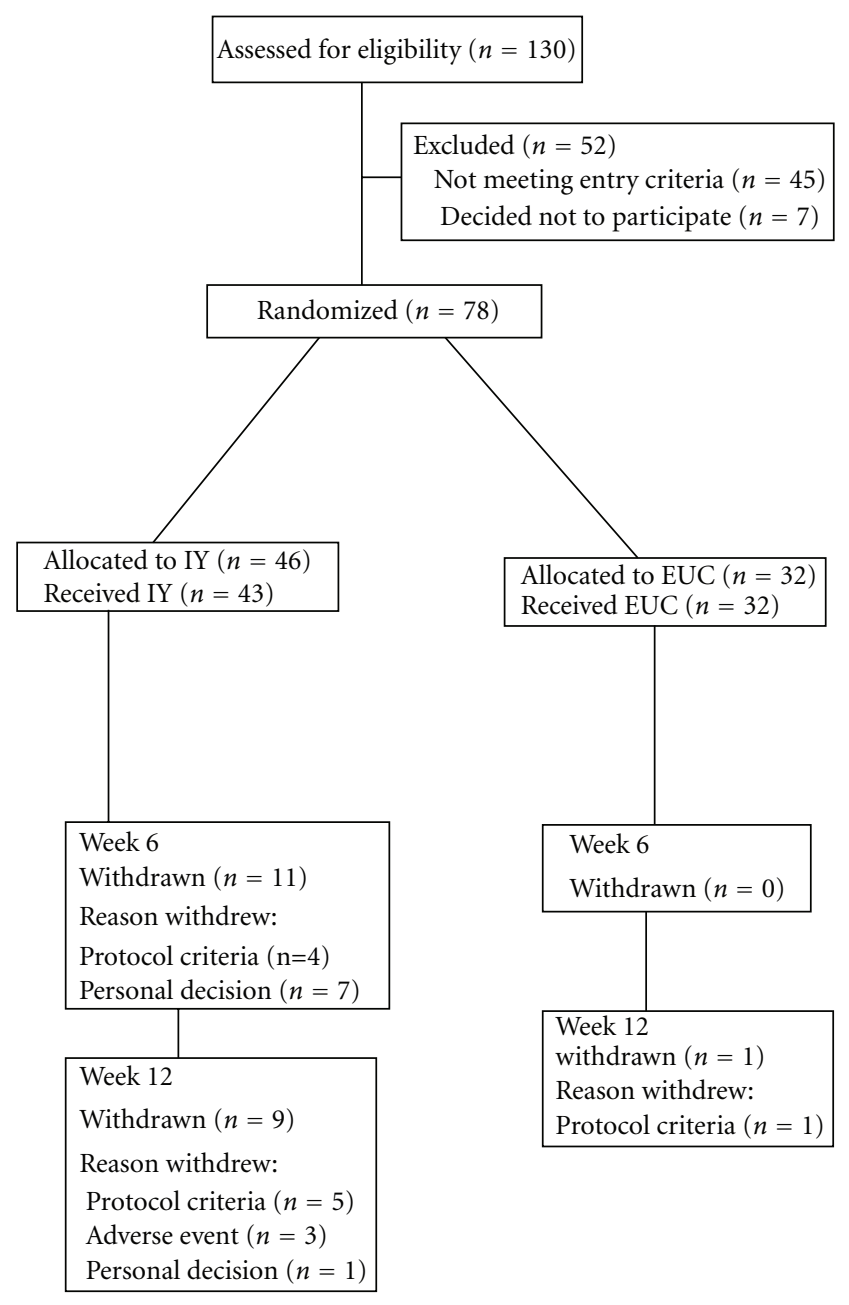

Figure 1: Study flow diagram.

Week 6 and between baseline and Week 12 for the IY arm and the EUC group. The standard $t$-test of differences in means assumes that the variances are equal. This assumption may be too strong. If violated, it may underestimate the true standard error and lead to $P$-values that are too small. We used Satterthwaite's formula to correct the standard error and $P$-values when needed.

\section{Results}

3.1. Study Participation and Retention. Figure 1 shows participant retention. Dropout rate exceeded the estimated 15\% in the IY arm. Because the dropout rate was imbalanced (zero in the EUC arm at this time), randomization was changed from $1: 1$ to $4: 1$ (IY to EUC) for the last cohort to try and balance group numbers.

3.2. Demographics. Baseline demographics of enrolled subjects are shown in Table 2. There were no significant baseline differences between those who dropped out or completed or between completers when comparing treatment groups. 
TABLE 2: Baseline demographics by treatment ${ }^{(a)}$.

\begin{tabular}{lcc}
\hline Characteristics & $\begin{array}{c}\text { IY } \\
(n=46)\end{array}$ & $\begin{array}{c}\text { EUC } \\
(n=32)\end{array}$ \\
\hline Male, $n$ & 23 & 16 \\
Female, $n$ & 23 & 16 \\
Race, $n$ & & \\
$\quad$ African American & 18 & 14 \\
$\quad$ Asian/native Hawaiian/Pacific Islander & 3 & 1 \\
$\quad$ Caucasian & 21 & 16 \\
$\quad$ Hispanic & 3 & 0 \\
$\quad$ Other & 1 & 1 \\
Age $($ years $)$ & $48.2 \pm 1.6$ & $48.3 \pm 2.4$ \\
Weight $(\mathrm{kg})$ & $86.4 \pm 3.1$ & $87.7 \pm 3.4$ \\
BMI $\left(\mathrm{kg} / \mathrm{m}^{2}\right)$ & $29.6 \pm 0.9$ & $30.5 \pm 1.0$ \\
SBP $(\mathrm{mmHg})$ & $140 \pm 1$ & $140 \pm 1$ \\
DBP $(\mathrm{mmHg})$ & $87 \pm 1$ & $86 \pm 1$ \\
\hline
\end{tabular}

${ }^{\text {(a) }}$ Results reported as mean (standard error).

3.3. Twenty-Four Hour AMBP. SBP and DBP of $24 \mathrm{~h}$ at Weeks 0,6 and 12 are available from 26 people in the IY group and 31 people in the EUC group, which is shown in Table 3. At 6 weeks, $24 \mathrm{~h} \mathrm{SBP}$ significantly improved in the EUC group when compared with the IY group ( $P$ value between groups $=.04$ ). There was no significant difference in $24 \mathrm{~h} \mathrm{DBP}$ at 6 weeks between groups and no difference in either $24 \mathrm{~h}$ SBP or DBP at 12 weeks between groups. Among IY participants, there was no significant change in $24 \mathrm{~h} \mathrm{SBP}$ or DBP at 6 weeks compared to baseline, but both measures improved at 12 weeks $(P=.05$ for SBP and $P<$ .01 for DBP). Within the EUC group, $24 \mathrm{~h} \mathrm{SBP}$ and DBP significantly decreased from baseline at 6 weeks $(P=.02$, SBP; $P<.05$, DBP), but changes in these measures at 12 weeks were no longer significant. This data is shown graphically in Figure 2.

3.4. Weight and BMI. There was no significant change in weight or BMI over time between groups. However, weight and BMI decreased significantly in the EUC group at 12 weeks compared to baseline. Weight decreased from $88.8 \pm$ $3 \mathrm{~kg}$ at Week 0 to $87.2 \pm 0.7 \mathrm{~kg}$ at Week 12 (with a change of $-1.6 \mathrm{~kg}, P=.02)$ in the EUC group. There was no change in weight in the IY group $(85.5 \pm 3.9 \mathrm{~kg}$ at Week 0 and $87.0 \pm 1.8 \mathrm{~kg}$ at Week 12 ; change of $1.5 \mathrm{~kg}, P=0.4)$. BMI also decreased significantly in the EUC group at 12 weeks $(P$ $<.05)$ but remained unchanged in the IY group at 12 weeks.

3.5. Diet Composition. The IY group did not receive any individualized dietary instructions or guidelines. There was a significant decrease in energy intake at 6 weeks in the EUC group compared to the IY group that was due to a decrease in total fat and carbohydrates. EUC participants also had a significant decrease in dietary sodium intake at 6 weeks compared to the IY group, yet sodium levels had started to revert towards baseline at 12 weeks. Urine sodium levels also showed a significant decrease at 6 weeks implying dietary compliance with a sodium restricted diet; however, at 12 weeks, urine sodium levels had increased and were no longer significant. At 12 weeks, the change in potassium intake was significantly greater in the EUC group when compared with the IY group.

3.6. Biochemical Measures. There were no differences over time between treatments in plasma levels of aldosterone, renin or metanephrines or in urinary or salivary cortisol concentrations. There were no significant changes in routine chemistry measures between groups at 12 weeks.

3.7. Psychometric Evaluations. There were no significant inter- or intra-group differences in psychometric data at 6 or 12 weeks for the POMS and PSS. However, the SF-36 health transition subscale was statistically significant for the EUC group at 6 weeks $(P=.02)$ but was not sustained at 12 weeks. Correlations between psychometric data and the change in $24 \mathrm{~h}$ SBP were found in PSS $(P=.03)$, POMS $(P=0.4)$ and Global scores $(P=.001)$ in both groups. There was a correlation between the change in the total SF-36 and change in salivary cortisol $(P=.02)$ for both groups (data not shown).

\section{Discussion}

This is the first randomized controlled clinical trial in USA assessing the effects of IY compared with dietary measures on $24 \mathrm{~h} \mathrm{ABPM}$ in patients with prehypertension or Stage I hypertension. Although we did not find that IY significantly improved any measured endpoint over the EUC group, our data demonstrated that IY produced clinically meaningful reductions in SBP and DBP at 12 weeks compared to baseline within the IY group. The mean $24 \mathrm{~h}$ SBP decreased by $6 \mathrm{mmHg}$ at 12 weeks compared to baseline. This is comparable to estimated reductions in SBP when following individual established LSM including weight loss, DASH diet, sodium reduction or physical activity [3] and was numerically greater than the change in mean $24 \mathrm{~h}$ SBP in the EUC group at 12 weeks. Data from a meta-analysis of individual data for 1 million adults from 61 prospective observational studies of BP and mortality demonstrated that even a small 2-mmHg fall in mean SBP was associated with a $7 \%$ lower risk of ischemic heart disease (IHD) death and a $10 \%$ lower risk of stroke death [18] emphasizing that even small reductions in SBP in large populations can have important cardiovascular morbidity and mortality benefits.

Psychological stress is a risk factor for hypertension [1921] and the Canadian Hypertension Society recognizes the role of stress management techniques in managing hypertension $[22,23]$. Reduction of stress using transcendental meditation has been shown to decrease BP in African American hypertensives $[24,25]$. Various forms of yoga also claim to reduce stress by integrating the body and mind. Although there are several published studies investigating the effects of various forms of yoga on hypertension [8, 9, $12,13,26]$, most of these were uncontrolled case reports 
TABLE 3: Changes in $24 \mathrm{~h} \mathrm{BP}$ parameters ${ }^{(\mathrm{a})}$.

\begin{tabular}{|c|c|c|c|c|c|c|}
\hline & \multicolumn{3}{|c|}{ IY } & \multicolumn{3}{|c|}{ EUC } \\
\hline & Week 0 & Week 6 & Week 12 & Week 0 & Week 6 & Week 12 \\
\hline $\mathrm{SBP}(\mathrm{mmHg})$ & $132(3)$ & $133(2)$ & $126(3)^{*}$ & $135(3)$ & $130(2)^{* *}$ & $131(2)$ \\
\hline DBP (mmHg) & $83(2)$ & $83(1)$ & $78(2)^{* * *}$ & $82(2)$ & $79(1)^{* *}$ & $80(1)$ \\
\hline MAP (mmHg) & $99(2)$ & $99(1)$ & $94(2)^{* *}$ & $99(2)$ & $96(1)^{* *}$ & $97(2)$ \\
\hline Heart rate $(\mathrm{mmHg})$ & $70(2)$ & $70(1)$ & $68(2)$ & $69(2)$ & $67(1)$ & $67(1)$ \\
\hline
\end{tabular}

(a) Results reported as mean (standard error).

${ }^{*} P=.05,{ }^{* *} P<.05,{ }^{* * *} P<.01$.

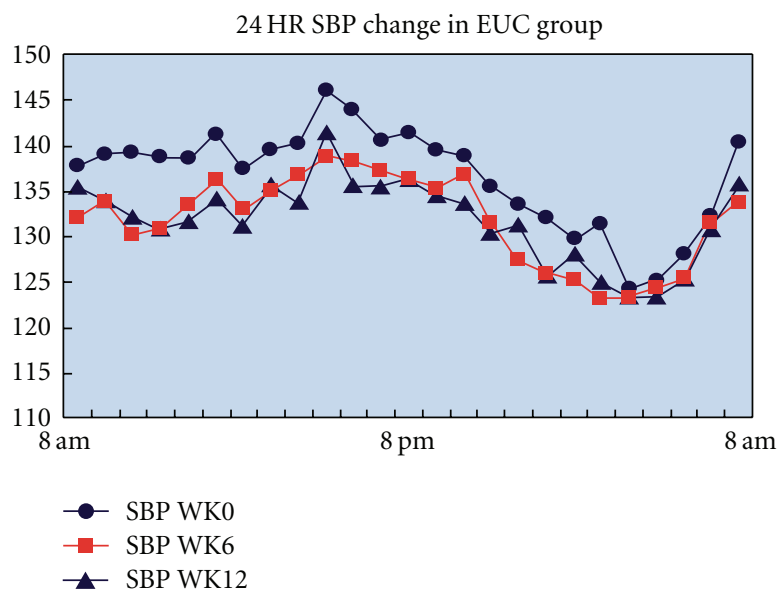

(a)

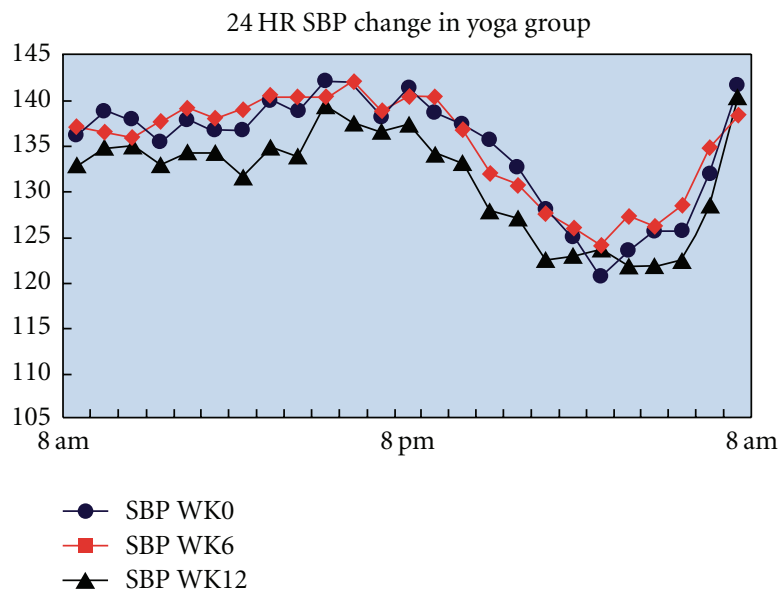

(c)

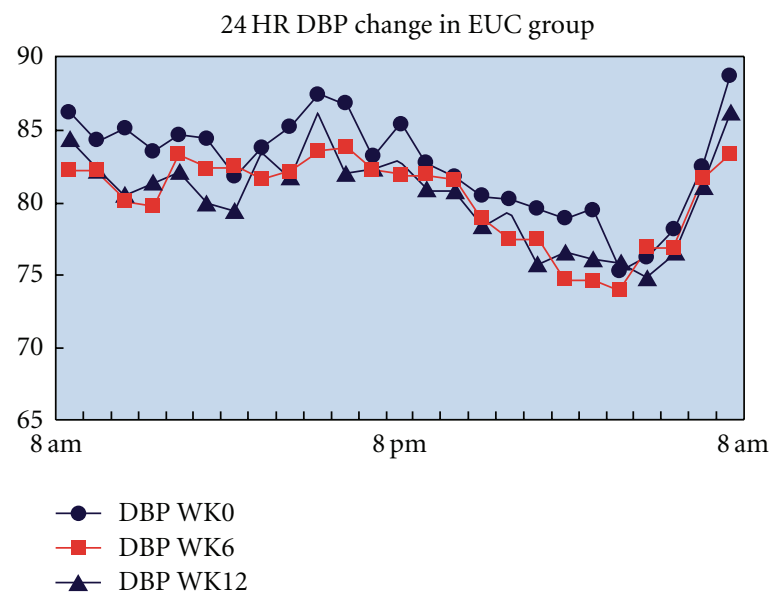

(b)

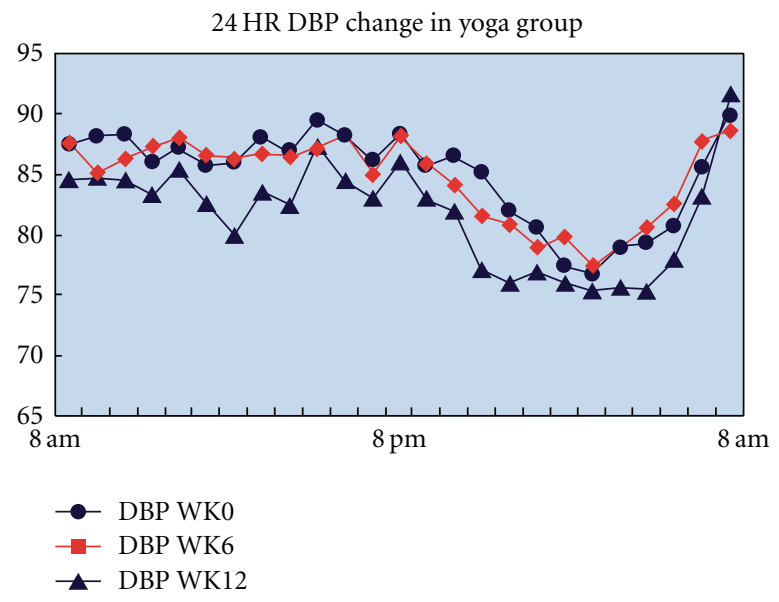

(d)

Figure 2: Changes in mean $24 \mathrm{~h} \mathrm{SBP}$ and DBP in the IY and EUC groups at randomization, 6 and 12 weeks.

or small cohort studies conducted in India with significant methodological limitations $[8,11,12]$. There are only three randomized controlled trials of any form of yoga for hypertension $[9,10,26]$. The most recent study was an 8week pranayama and asana yoga program conducted in 27 untreated hypertensive patients and 27 controls in Thailand [26]. The experimental group significantly reduced SBP by $24 \mathrm{mmHg}$ at 8 weeks compared to $2 \mathrm{mmHg}$ increase in the control group. DBP significantly improved by $18 \mathrm{mmHg}$ in the experimental group compared to an increase of
$2 \mathrm{mmHg}$ in the control group. Details regarding methods and accuracy of BP measurement were not included. In India, 33 hypertensive adults were randomly assigned to three groups (yoga, medications only, or no therapy) and were followed for 11 weeks [9]. Yoga was performed at home for $6 \mathrm{~h}$ per week; at the end of the study, SBP reduced by an impressive $33 \mathrm{mmHg}$ compared with $4 \mathrm{mmHg}$ in the control group and $24 \mathrm{mmHg}$ in the poorly described drug therapy group. The differences were significant compared with both control and drug treatment. In an older RCT 
from England, 43 patients with known hypertension, most of whom were already medically treated, were randomized to yoga plus biofeedback or usual care [10]. Treatment reduced SBP by $26 \mathrm{mmHg}$ versus $9 \mathrm{mmHg}$ in the control $(P<$ .005). However, this study used a mixed intervention which included biofeedback in addition to yoga, which was more like TM and did not include any movement.

There are several limitations that can affect interpretation of the data. Subjects enrolled in the trial were possibly more likely than the general population to be motivated to pursue a lifestyle modification such as yoga as a therapy for hypertension and this can lead to a self-selected study population being enrolled in the trial. Although randomized, the trial was unblinded and though we included the EUC control group, it was an active control. While blinding is ideal to eliminate measurement bias, it is not feasible with behavioral interventions. The EUC intervention produced significant changes in some BP parameters and although numerically smaller than the IY group, at 12 weeks we were underpowered to detect a statistically significant difference between groups. The lack of differences between groups may be attributed to both the unanticipated high dropout rate in the IY group and the duration of the trial which was probably too short to detect greater changes in the IY group as it is apparent that changes in BP in the IY group took longer to occur.

The EUC group produced a significant reduction in $24 \mathrm{~h}$ SBP at 6 weeks compared to the IY group as well as a significant within group improvement in DBP. While these variables were not statistically significant at 12 weeks either between groups or within the EUC group, they were still clinically meaningful and met the primary aim of decreasing SBP by $6 \mathrm{mmHg}$ from baseline. Changes in $24 \mathrm{~h} \mathrm{SBP}$ and DBP were comparable to changes seen in previous clinical trials assessing the effects of dietary modifications alone [27, 28 ] and in combination with other lifestyle modifications on BP [29]. Body weight in EUC-randomized subjects decreased significantly within this group at 12 weeks compared to baseline. EUC participants made significant improvements in energy, fat, carbohydrates and sodium intake at 6 weeks, yet all variables started to revert towards baseline at 12 weeks. This was seen particularly with urine sodium which showed a significant decrease at 6 weeks implying dietary compliance with a sodium-restricted diet; however, at 12 weeks, urine sodium levels had increased and were no longer significant indicating difficulty in maintaining dietary sodium restriction. These findings are supported by others revealing that dietary recommendations are hard to follow over time [6].

Another limitation is that the two intervention groups met with unequal frequency and this can introduce confounding. Due to the high attrition rate in the IY group which exceeded expectations, a change was necessitated in the randomization scheme for the last cohort from $1: 1$ to 4 : 1 (IY: EUC). Although we acknowledge this as a limitation, there were no differences in the baseline demographics in the IY group in the subjects who dropped out compared to the subjects who completed. Twenty participants (43\%) randomized to IY did not complete, thereby reducing the numbers of completers and thus the power of the trial. Of the IY non completers, $60 \%$ were due to not being able to adhere to the class schedule. This may be due to several reasons. IY is not an aerobic form of exercise like other more Westernized forms of yoga, and subjects recruited into the study were "yoga-naïve" and may have expected a more traditional type of yoga and may have been discouraged by the rigid style of our IY program. The yoga program was also designed as "top heavy" program which decreased in intensity over the trial. Yoga practice for naïve yoga subjects requires slow transition with increase in intensity of the yoga program over a few months.

We sought to determine the role of reductions in stress as a mechanism for the BP pressure changes in our subjects using questionnaires and salivary cortisol. Our failure to find such association in the IY group was disappointing. Other studies evaluating yoga practice suggests that yoga may reduce oxidative stress [30] and endothelial-dependent vasodilatation in patients with established coronary artery disease [16]. Additional studies are needed incorporating measures of oxidative stress and endothelial function.

Our data demonstrates that the effects of IY on BP at 12 weeks as assessed by $24 \mathrm{~h} \mathrm{ABPM}$ were comparable or greater than that produced by an EUC intervention in adults with prehypertension and stage 1 hypertension. This pilot has provided useful data and experience that we can use for future studies. Specifically, when designing a future trial, we would include three groups and assess the combination of EUC and yoga versus intervention alone, we would enroll subjects for a longer duration (24 weeks) to detect greater, more sustained reductions in BP in the yoga groups. We would redesign the yoga program with specific attention to improving adherence and gradually increasing yoga practice over the course of the trial. We would also consider using a less rigid, more aerobic type of yoga that is more available and accessible to the public and therefore more likely to be incorporated into patients' lifestyles as a long-term lifestyle adjustment. However, given the general safety profile of yoga and general public appeal, we feel it is important to fund additional studies in order to establish the long-term efficacy and the potential mechanisms in BP reductions associated with yoga therapy in patients with prehypertension and Stage 1 hypertension.

\section{Funding}

National Institutes of Health grants R21AT002353-02 (to P.O.S. and R.R.T.) and M01-RR00040 (General Clinical Research Center) and National Center for Complementary and Alternative Medicine (NCCAM).

\section{Acknowledgments}

The authors thank the participants for their participation in this trial. They would also like to thank Sue Yang-Novelino for coordinating the study and IY Instructors, Joan White, Elzbeita Jablonska and Russ Taylor, for conducting the IY classes. They would also like to thank Jianing Yang and Daniel 
Polsky for programming assistance, Lisa Basel-Brown for nutrient analysis and the GCRC nurses for their help with patient care.

\section{References}

[1] L. E. Fields, V. L. Burt, J. A. Cutler, J. Hughes, E. J. Roccella, and P. Sorlie, "The burden of adult hypertension in the United States 1999 to 2000: a rising tide," Hypertension, vol. 44, no. 4, pp. 398-404, 2004.

[2] I. Hajjar and T. A. Kotchen, "Trends in prevalence, awareness, treatment, and control of hypertension in the United States, 1988-2000," Journal of the American Medical Association, vol. 290, no. 2, pp. 199-206, 2003.

[3] A. V. Chobanian, G. L. Bakris, H. R. Black et al., "Seventh report of the joint national committee on prevention, detection, evaluation, and treatment of high blood pressure," Hypertension, vol. 42, no. 6, pp. 1206-1252, 2003.

[4] R. S. Vasan, M. G. Larson, E. P. Leip et al., "Impact of highnormal blood pressure on the risk of cardiovascular disease," The New England Journal of Medicine, vol. 345, no. 18, pp. 1291-1297, 2001.

[5] A. V. Chobanian, G. L. Bakris, H. R. Black et al., "The seventh report of the joint national committee on prevention, detection, evaluation, and treatment of high blood pressure: the JNC 7 report," Journal of the American Medical Association, vol. 289, no. 19, pp. 2560-2572, 2003.

[6] V. J. Stevens, E. Obarzanek, N. R. Cook et al., "Long-term weight loss and changes in blood pressure: results of the trials of hypertension prevention, phase II," Annals of Internal Medicine, vol. 134, no. 1, pp. 1-11, 2001.

[7] D. Cohen and R. R. Townsend, "Yoga and hypertension," The Journal of Clinical Hypertension, vol. 9, pp. 800-801, 2007.

[8] A. Damodaran, A. Malathi, N. Patil, N. Shah, S. , and S. Marathe, "Therapeutic potential of yoga practices in modifying cardiovascular risk profile in middle aged men and women," Journal of Association of Physicians of India, vol. 50, no. 5, pp. 633-640, 2002.

[9] R. Murugesan, N. Govindarajulu, and T. K. Bera, "Effect of selected yogic practices on the management of hypertension," Indian Journal of Physiology and Pharmacology, vol. 44, no. 2, pp. 207-210, 2000.

[10] C. Patel, "12-month follow up of yoga and bio feedback in the management of hypertension," The Lancet, vol. I, no. 7898, pp. 62-64, 1975.

[11] C. Lakshmikanthan, R. Alagesan, S. Thanikachalam et al., "Long term effects of yoga on hypertension and/or coronary artery disease," The Journal of the Association of Physicians of India, vol. 27, no. 12, pp. 1055-1058, 1979.

[12] T. H. Tulpule, H. M. Shah, S. J. Shah, and H. K. Haveliwala, "Yogic exercises in the management of ischaemic heart disease," Indian Heart Journal, vol. 23, no. 4, pp. 259-264, 1971.

[13] K. K. Datey, S. N. Deshmukh, C. P. Dalvi, and S. L. Vinekar, "'Shavasan": a yogic exercise in the management of hypertension," Angiology, vol. 20, no. 6, pp. 325-333, 1969.

[14] J. Granath, S. Ingvarsson, U. von Thiele, and U. Lundberg, "Stress management: a randomized study of cognitive behavioural therapy and yoga," Cognitive Behaviour Therapy, vol. 35, no. 1, pp. 3-10, 2006.

[15] L. E. Carlson, M. Speca, P. Faris, and K. D. Patel, "One year pre-post intervention follow-up of psychological, immune, endocrine and blood pressure outcomes of mindfulnessbased stress reduction (MBSR) in breast and prostate cancer outpatients," Brain, Behavior, and Immunity, vol. 21, no. 8, pp. 1038-1049, 2007.

[16] S. Sivasankaran, S. Pollard-Quintner, R. Sachdeva, J. Pugeda, S. M. Hoq, and S. W. Zarich, "The effect of a six-week program of yoga and meditation on brachial artery reactivity: do psychosocial interventions affect vascular tone?" Clinical Cardiology, vol. 29, no. 9, pp. 393-398, 2006.

[17] E. O'Brien, F. Mee, N. Atkins, and K. O’Malley, “Accuracy of the SpaceLabs 90207 determined by the British Hypertension Society Protocol," Journal of Hypertension, vol. 9, no. 6, pp. 573-574, 1991.

[18] S. Lewington, R. Clarke, N. Qizilbash, R. Peto, and R. Collins, "Age-specific relevance of usual blood pressure to vascular mortality: a meta-analysis of individual data for one million adults in 61 prospective studies," The Lancet, vol. 360, no. 9349, pp. 1903-1913, 2002.

[19] S. Levenstein, M. W. Smith, and G. A. Kaplan, "Psychosocial predictors of hypertension in men and women," Archives of Internal Medicine, vol. 161, no. 10, pp. 1341-1346, 2001.

[20] J. H. Markovitz, K. A. Matthews, W. B. Kannel, J. L. Cobb, and R. B. D'Agostino, "Psychological predictors of hypertension in the Framingham study: is there tension in hypertension?" Journal of the American Medical Association, vol. 270, no. 20, pp. 2439-2443, 1993.

[21] T. Rutledge and B. E. Hogan, "A quantitative review of prospective evidence linking psychological factors with hypertension development," Psychosomatic Medicine, vol. 64, no. 5, pp. 758-766, 2002.

[22] J. D. Spence, P. A. Barnett, W. Linden, V. Ramsden, and P. Taenzer, "Lifestyle modifications to prevent and control hypertension. 7. Recommendations on stress management. Canadian Hypertension Society, Canadian Coalition for High Blood Pressure Prevention and Control, Laboratory Centre for Disease Control at Health Canada, Heart and Stroke Foundation of Canada," Canadian Medical Association Journal, vol. 160, no. 9, pp. S46-50, 1999.

[23] N. A. Khan, B. Hemmelgarn, R. Padwal et al., "The 2007 Canadian Hypertension Education Program recommendations for the management of hypertension: part 2: therapy," Canadian Journal of Cardiology, vol. 23, no. 7, pp. 539-550, 2007.

[24] R. H. Schneider, F. Staggers, C. N. Alexander et al., "A randomized controlled trial of stress reduction for hypertension in older African Americans," Hypertension, vol. 26, no. 5, pp. 820-827, 1995.

[25] R. H. Schneider, C. N. Alexander, F. Staggers et al., "A randomized controlled trial of stress reduction in African Americans treated for hypertension for over one year," American Journal of Hypertension, vol. 18, no. 1, pp. 88-98, 2005.

[26] R. McCaffrey, P. Ruknui, U. Hatthakit, and P. Kasetsomboon, "The effects of yoga on hypertensive persons in Thailand," Holistic Nursing Practice, vol. 19, no. 4, pp. 173-180, 2005.

[27] L. J. Appel, T. J. Moore, E. Obarzanek et al., "A clinical trial of the effects of dietary patterns on blood pressure," The New England Journal of Medicine, vol. 336, no. 16, pp. 1117-1124, 1997.

[28] F. M. Sacks, L. P. Svetkey, W. M. Vollmer et al., "Effects on blood pressure of reduced dietary sodium and the dietary approaches to stop hypertension (DASH) diet," The New England Journal of Medicine, vol. 344, no. 1, pp. 3-10, 2001.

[29] L. J. Appel, C. M. Champagne, D. W. Harsha, L. S. Cooper, E. Obarzanek, P. J. Elmer et al., "Effects of comprehensive lifestyle modification on blood pressure control: main results 
of the PREMIER clinical trial," Journal of the American Medical Association, vol. 289, pp. 2083-2093, 2003.

[30] R. K. Yadav, R. B. Ray, R. Vempati, and R. L. Bijlani, "Effect of a comprehensive yoga-based lifestyle modification program on lipid peroxidation," Indian Journal of Physiology and Pharmacology, vol. 49, no. 3, pp. 358-362, 2005. 


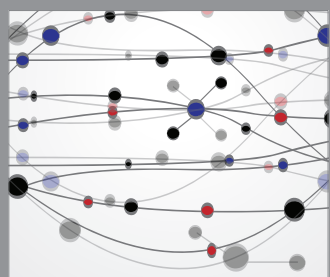

The Scientific World Journal
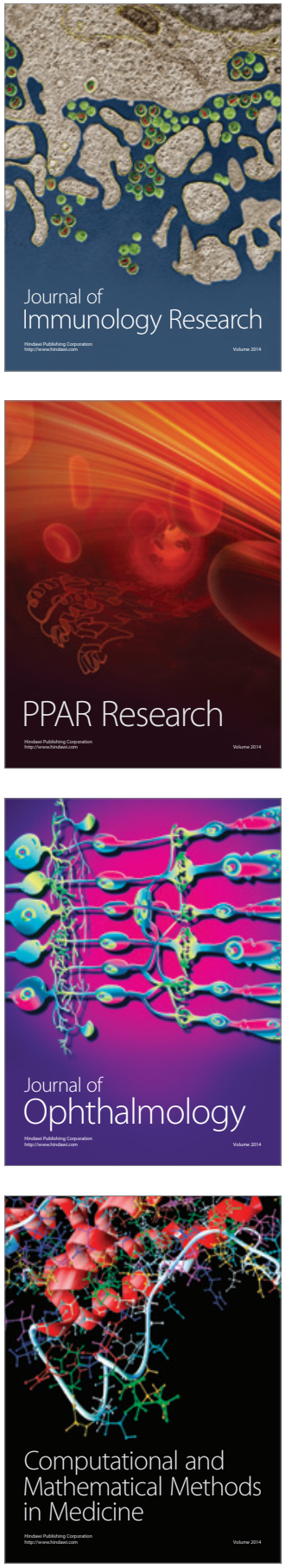

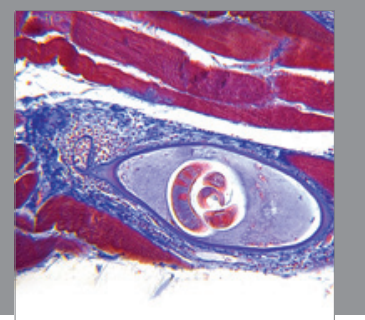

Gastroenterology

Research and Practice
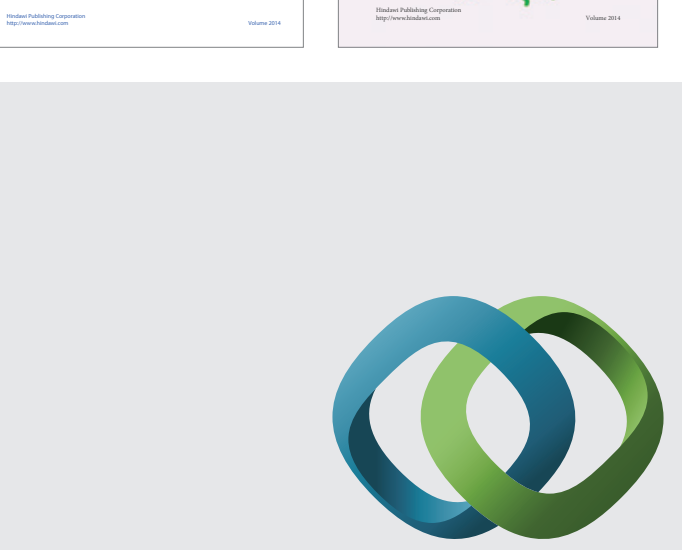

\section{Hindawi}

Submit your manuscripts at

http://www.hindawi.com
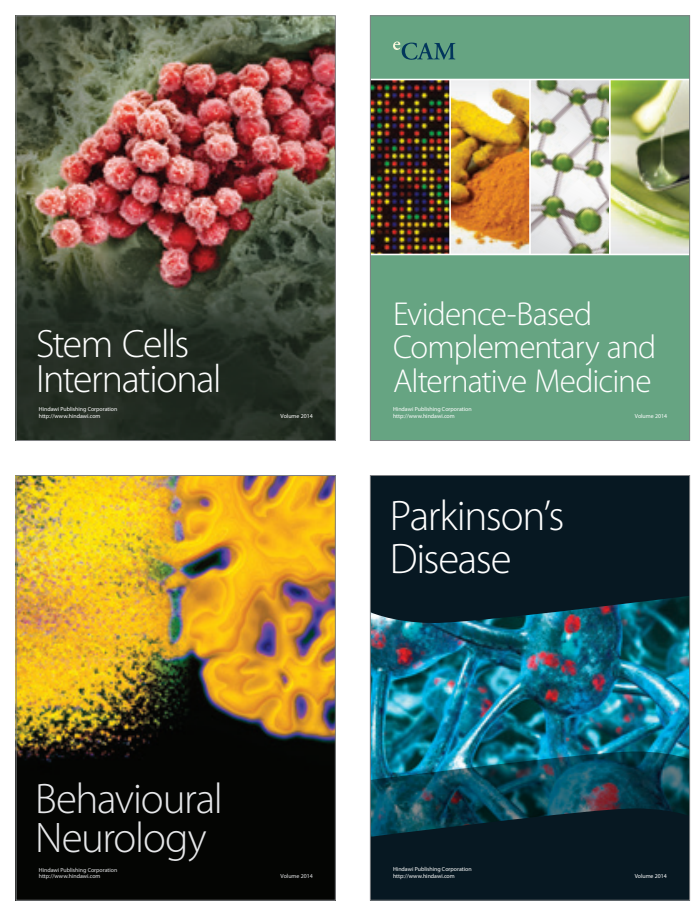

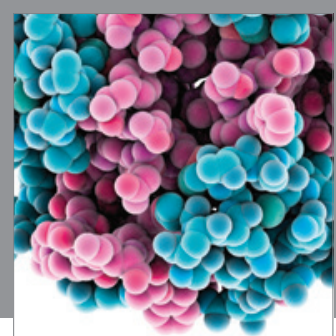

Journal of
Diabetes Research

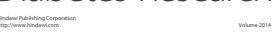

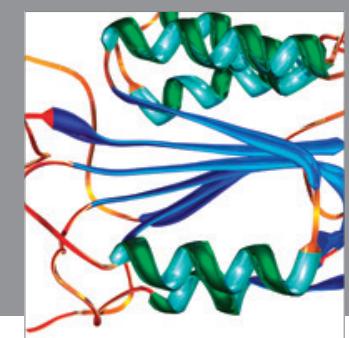

Disease Markers
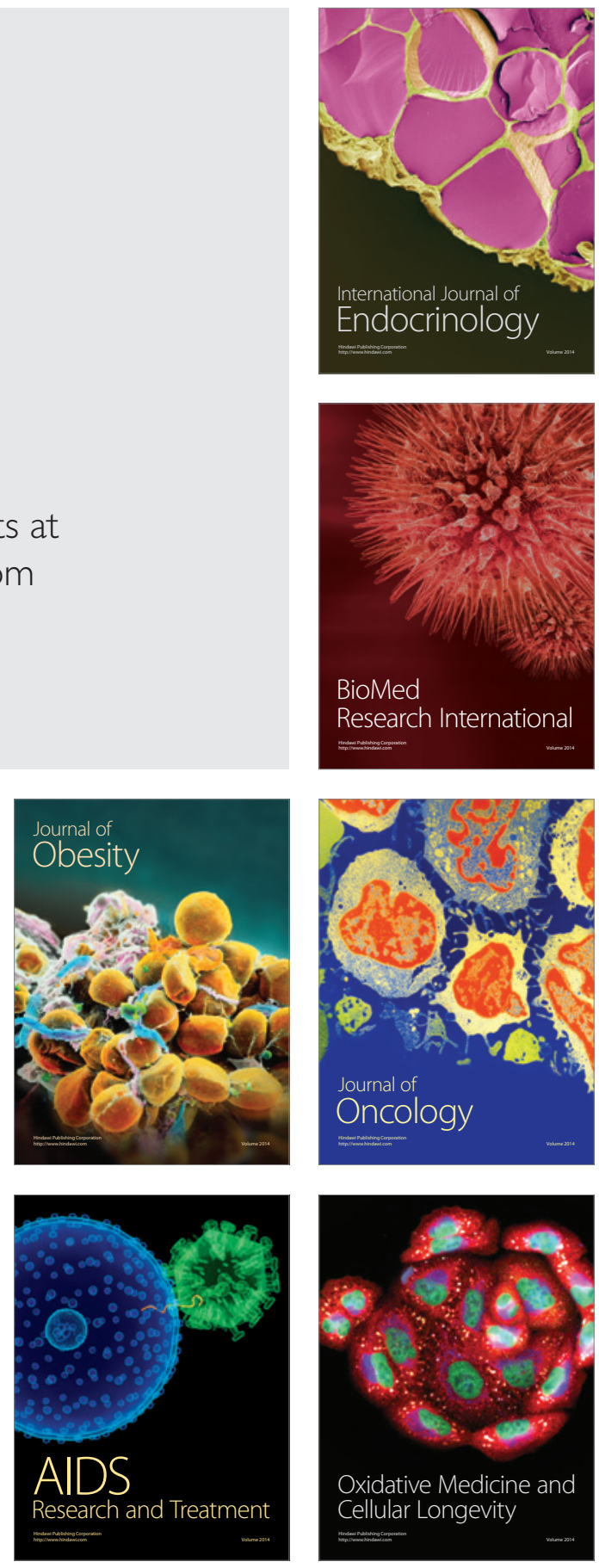